\title{
ON CERTAIN SYSTEMS WHICH ARE ALMOST GROUPS
}

HENRY B. MANN

We consider systems $\mathfrak{S}$ of elements $A, B, \ldots$ satisfying the following postulates.

I. To every pair of elements $A, B$ in $\subseteq$ there exists a uniquely determined product $A \cdot B$ in $\subseteq$.

II. $(A B) C=A(B C)$.

III. There exists in $\subseteq$ an element $E$ such that $E A=A$ for every $A$.

IV. To every $A$ in $\subseteq$ there exists $a n A^{\prime}$ in $\subseteq$ such that $A A^{\prime}=E$.

The system $\subseteq$ differs from a group only in that it contains a left unit and right inverse instead of a right unit and right inverse. We shall call such a system a left right system, abbreviated $(l, r)$ system.

Definition 1. An element $F$ is called an idempotent if $F^{2}=F$.

Proposition 1. If $F$ is idempotent then $F E=E$.

Proof. From $F F=F$ we have $F F F^{-1}=F F^{-1}$, hence $F E=E$.

Proposition 2. If $F E=E$ then $F$ is idempotent.

For $F F=F E F=E F=F$.

PROPOsition 3. The idempotents of $a(l, r)$ system form $a(l, r)$ system.

Proof. If $F$ and $F^{\prime}$ are idempotent then by Proposition 1

$$
F F^{\prime} E=F E=E,
$$

hence $F F^{\prime}$ is idempotent and postulate I is fulfilled. Postulate II is fulfilled since it is fulfilled in $\mathfrak{S}$. $E$ itself is idempotent and is by Proposition 1 a right inverse to every idempotent. Hence all the postulates of a $(l, r)$ system are fulfilled.

Proposition 4. If $F$ is idempotent and $A$ is any element then $F A=A$.

For $F A=F E A=E A=A$.

Definition 2. $A(l, r)$ system which contains only idempotents will be called an idempotent $(l, r)$ system.

From Proposition 4 we see that in every idempotent $(l, r)$ system every product is equal to its last factor. On the other hand it is easy to verify that every system of elements in which every product is

Received by the editors March 18, 1944. 
equal to its last factor is an idempotent $(l, r)$ system since every element of such a system can be considered the unit element and is then inverse to all elements. From the foregoing we have the following theorem.

THEOREM 1. There exists one and only one idempotent $(l, r)$ system of order $n\left(n=1,2, \cdots\right.$ ad inf.). It consists of the elements $A_{1}, A_{2}, \cdots, A_{n}$ with the law of composition $A_{i} A_{j}=A_{j}$.

Remark. Similarly one and only one idempotent $(l, r)$ system exists for every cardinal number.

PRoposition 5. The elements of any $(l, r)$ system which are of the form $A F$ where $F$ is a fixed idempotent form a group.

Proof. $(A F)(B F)=A(F B) F=A B F$ by Proposition 4 . The composition is by hypothesis associative, $F$ is a right unit and $A^{-1} F$ a right inverse. Since the system forms a group we must have $A^{-1} F A F=F$.

Let $\Im=\left(E, \quad F_{2}, \cdots\right)$ be an idempotent $(l, r)$ system, $\$$ $=\left(\bar{E}, A_{2}, \cdots\right)$ a group. We form a system $\subseteq$ in the following way: S consists of all pairs $A_{i} F_{3}$ with the following rule of combination:

$$
A_{i} F_{j} A_{k} F_{l}=A_{i} A_{k} F_{l} .
$$

It is easy to verify that $\subseteq$ is a $(l, r)$ system.

DeFinition 3. The system 5 defined in the preceding paragraph is called the direct product of $\Im$ and $\$$ S.

PROposition 6. Every element of $a(l, r)$ system may be written in the form $(A E) F$ where $F$ is idempotent.

Proof. We have $\left(A^{-1} A\right)\left(A^{-1} A\right)=A^{-1}\left(A A^{-1}\right) A=A^{-1}(E A)=A^{-1} A$. Hence $A^{-1} A$ is idempotent. But $A=A A^{-1} A=A\left(E A^{-1} A\right)=A E\left(A^{-1} A\right)$.

Proposition 7. If $A E F=B E F^{\prime}$ where $F$ and $F^{\prime}$ are idempotents then $A E=B E$ and $F=F^{\prime}$.

Proof. From $A E F=B E F^{\prime}$ we have $A E F E=B E F^{\prime} E$. Hence on account of Proposition 1, $A E=B E$. Since the elements of the form $A E$ form a group, $A^{-1} E$ is a left inverse of $A E$ and $F=F^{\prime}$ follows.

From Propositions 5, 6 and 7 we have the following theorem.

THEOREM 2. Every $(l, r)$ system is the direct product of an idempotent $(l, r)$ system and a group.

Proposition 8. Every idempotent $F$ of $a(l, r)$ system $\sqrt{5}$ is a unit element of $\mathfrak{S}$. 
For by Proposition 4, $F A=A$ for every $A$ and $A\left(A^{-1} F\right)=E F=F$.

Let $S_{1}, \cdots, S_{n}$ be $n$ statements. Let $A_{i}$ be the statement: "All the preceding statements are annulled but $S_{i}$ is true." It is interesting to note that the statements $A_{i}$ form an idempotent $(l, r)$ system.

Ohio State University

\section{A CONJECTURE IN ELEMENTARY NUMBER THEORY}

\section{LEON ALAOGLU AND PAUL ERDÖS}

A well known conjecture of Catalan states that if $f(n)$ is the sum of all divisors of $n$ except $n$, then the sequence of iterates of $f(n)$ is either eventually periodic or ends at 1 . It not only seems impossible to prove this, but it is also very difficult to verify. ${ }^{1}$

Another conjecture of Poulet, ${ }^{2}$ which appears equally difficult to prove, has the doubtful merit that it is easy to verify. Let $\sigma(n)$ be the sum of all divisors of $n$, and let $\phi(n)$ be Euler's function. Then for any integer $n$ the sequence

$$
f_{0}(n)=n, \quad f_{2 k+1}(n)=\sigma\left(f_{2 k}(n)\right), \quad f_{2 k}(n)=\phi\left(f_{2 k-1}(n)\right)
$$

is eventually periodic.

We have verified this conjecture to $n=10000$ (extending Poulet's verification) by using Glaisher's tables. ${ }^{3}$ The checking was facilitated by the following observation: if the conjecture is to be checked for all $n<x$, it is enough to find a member of the sequence other than the first which is less than $x$.

The longest cycle found was in the sequence $f_{k}(9216)$. It starts with $f_{6}(9216)$, and is: $34560,122640,27648,81800,30976,67963,54432$, $183456,48384,163520,55296,163800,34560$. However our method of checking does not show that this is the largest cycle up to 10000 , and in fact Poulet found that $f_{k}(1800)$ has the same length 12.

As a rule $\phi(\sigma(n))$ is less than $n$. In fact, it can be shown that for every $\epsilon>0, \phi(\sigma(n))<\epsilon n$, except for a set of density 0 . The proof follows from the following two observations:

Received by the editors May 18, 1944.

${ }^{1}$ L. E. Dickson, Theorems and tables on the sum of the divisors of a number, Quart. J. Math. vol. 44 (1913) pp. 264-296, and P. Poulet, La Chasse aux Nombres, vol. 1, pp. 68-72, and vol. 2, p. 188.

2 P. Poulet, Nouvelles suites arithmetiques, Sphinx vol. 2 (1932) pp. 53-54.

${ }^{3} \mathrm{~J}$. W. L. Glaisher, Number-divisor tables, British Association for the Advancement of Science, Mathematical Tables, vol. 8. 